(c) 2016 IEEE. Personal use of this material is permitted. Permission from IEEE must be obtained for all other uses, in any current or future media, including reprinting/republishing this material for advertising or promotional purposes, creating new collective works, for resale or redistribution to servers or lists, or reuse of any copyrighted component of this work in other works.

Digital Object Identifier (DOI): 10.1109/ETFA.2016.7733495

IEEE 21st International Conference on Emerging Technologies and Factory Automation (ETFA), Berlin, Germany, 2016

Control and communication in the Smart Transformer-fed grid

Zhi-Xiang Zou

Giampaolo Buticchi

Marco Liserre

Suggested Citation

Z. Zou, G. Buticchi and M. Liserre, "Control and communication in the Smart Transformer-fed grid," IEEE 21st International Conference on Emerging Technologies and Factory Automation (ETFA), Berlin, 2016, pp. 1-9. 


\title{
Control and Communication in the Smart Transformer-fed Grid
}

\author{
Zhi-Xiang Zou, Giampaolo Buticchi, and Marco Liserre \\ Chair of Power Electronics \\ Christian-Albrechts-Universitt zu Kiel \\ Kiel D-24143, Germany \\ Email: zz, gibu, ml@tf.uni-kiel.de
}

\begin{abstract}
The Smart Transformer (ST) is a solid-state transformer performing not only a voltage step-down function but also energy and information management. With the help of control and communication technology, the ST can increase hosting capacity of renewable energies and provide ancillary services to utility and customers. To better exploit the potential of a STfed grid, a proper design of architecture, control scenarios and its corresponding communication network has to be done. This paper aims at giving a better understanding of the functionality of the ST-fed grid and of the associated communication challenges.
\end{abstract}

Index Terms-Solid-state transformer, smart transformer, distribution grid, microgrid, control scenario, communication.

\section{INTRODUCTION}

A major concern for the electric engineering community is the increasing penetration of Distributed Energy Resources (DERs) and Electric Vehicles (EVs). In Germany for example, the share of electricity produced from DERs is about $30 \%$ in 2015 and the government was working towards an ambitious plan that aims to achieve $80 \%$ share of gross electricity production in 2050 [1]. The increase of DERs and EVs challenges the hosting capacity of the grid and arises technical and economical problems. From a energy management standpoint, power flow in a distribution grid is utterly changed by the power injection of DERs and EVs, modifying node/bus voltages and energy losses. This impacts the power system in terms of planning, operation, and protection [2], [3]. The intermittent nature of DERs and EVs further aggravates the severity of energy management, increasing the complexity of power control and power flow optimization. On the other hand, higher level of DER penetration introduces considerable power electronics devices which can incur potential harmonic distortion [4]. Identified by the HARMONY project [5], wideband harmonics ranging from medium frequency to high frequency have been brought into the grid and can be propagated to other grids (e.g. MV grid) through the transformers, which are likely to damage or shorten the lifetime of the equipments, cause unintentional islanding, and increase losses. Moreover, from a system modeling point of view, the nodal admittance matrix is drastically changed because of a vast of gridinterfaced converters [6]. Usually, this is manifested by the grid resonances which lead to harmonic stability [7].

978-1-5090-1314-2/16\$31.00 (c) 2016 IEEE
To address the aforementioned issues, exploratory concepts and research work are being investigated and demonstrated in some famous pilot projects, such as the FREEDM project [8], CERTS Microgrid [9], and BCIT RD\&D Grid [10], aim at enhancing generation, transfer, and store of the wide spread and variety of energy resources in a distribution grid or microgrid. In the context of smart grid [11], smart metering and communication technologies are extensively utilized in these projects, offering online monitoring, computing, diagnostic, and optimization, with the aim of introducing features like self-healing. Most of the mentioned projects proposed a wide use of power-electronics-based communication-less distributed control of the electric grid.

The Smart Transformer concept, first proposed by the HEART project [12], proposed instead to update the distribution transformers to solid-state transformers that can use communication to control the DER leading to a semi-decentralized management of the grid. This paper addresses the control and communication challenges of the smart transformer. To better understand the concept of ST-fed grid, a brief introduction and description of the system is given in Section II; in Section III, the possible control scenarios for a ST-fed grid is reviewed; in Section IV, the available communication technologies are presented and the impact of communication uncertainty is discussed. Finally, conclusions are drawn in Section V.

\section{ST-FED GRID CONCEPT}

\section{A. Centralized or Decentralized Control of Active Electric Grids}

The control of the active electric grid can be categorized as centralized and decentralized. The notion of centralized control requires a central controller to have full visibility of the grid and provide set-points to the distributed appliances. Meanwhile, historical data and online analysis of grid are demanded to assist distributed appliances better understanding grid condition. The architecture of a centralized control is usually with a hierarchical control structure, and one successful paradigm is the Multi-Agent System (MAS) for microgrid operation [13]. The primary feature of MAS is that the agents (e.g. Advanced Metering Infrastructure (AMI)) build the bridge between the top layer: for example Microgrid Central Controller (MGCC) and the bottom layer: distributed appliances. Being the core component in the MAS, agents are capable of data acquisition 
and restricted decision making, characterized by a certain level of autonomy. With the evolution of computation technology, rather than a medium exchanging information between two layers, agents gain more authorities and can independently make decisions to satisfy certain objective even without the permission of central controller. A contemporary MAS with the objective of explicit power control has been applied to a CIGRÉ low voltage benchmark micro-grid [14]. The agents obtain comprehensive knowledge of its subordinate network which can be either an entire micro-grid or a bunch of distributed appliances. Only an abstract view will be advertised from the agents to its superior layer in the forms of $P Q t$ profile, virtual cost, and belief function of local network. Receiving the responses from central controller, detailed setpoints and control instructs will be autonomously interpreted by the agents and sent to each appliance [15]. It is worth noticing that the autonomy level of agents is determined by several factors in terms of system scale, computation capability, and grid requirements. For example, in case of the small-scale isolated grid application with stringent power quality requirement, the central controller can be configured as a solo leader to communicate distributed appliances through AMI and take the full charge of control and incidents in the grid.

In contrast to the centralized control, decentralized control allows distributed appliances regulating themselves by distributed control strategies. Speaking of distributed control, it is always reminiscent of the communication-less control or the communication network with limited bandwidth. This is due to the outdated assumptions of limited communication infrastructures [16], [17]. One of the most popular communicationless distributed control in power system is droop control, which is widely used in the distribution grid and microgrid [18], [19]. Nevertheless, referring to the applications of Distributed Control System (DSC), a reliable communication network is essential for monitoring, command, and interaction between appliances. Moreover, in the context of smart grid, the electric grid requires sufficient communication infrastructures to achieve better performance and provide customized services to both MV and LV grids. A promising alternative is to use consensus algorithms to cooperatively control a large number of distributed appliances [20]. Based on Graph Theory, each distributed appliance interacts with neighboring nodes through communication network and appropriate set-points can be essentially established [21]. Necessary adjustment to the setpoints (e.g. secondary and tertiary control) can be carried out by the central controller, enabling a highly resilient grid.

Current trends of penetration of renewable are pushing the grid architecture from the highly centralized system to a more decentralized system [22]. One may notice that almost all the distributed appliances today are equipped with microprocessors, enabling them having higher self-control, computation and information exchange ability. In addition, the objectives of the modern grid become more diverse, not only characterized by energy planning, operation and harvest, but also with information acquisition, transmission and processing. It is becoming more and more inefficient and costly to integrate all the functionalities in one control center (e.g. SCADA) meeting the diverse and dynamically changing demands of customers.

\section{B. ST-fed Grid Paradigm}

A benchmark ST-fed grid is shown in Fig.1, in which a ST is being interconnected to the MV grid in parallel with other two distribution transformers. Being a three-stage solidstate transformer, the MV side and LV side of ST are decoupled, which essentially block the propagation of wideband harmonics and disturbance from one grid to the other. From the benchmark grid, it is seen that the ST offers diverse standard electric interfaces so that various types of distributed appliances like DERs, EVs, DC/AC loads can be flexibly coupled with different grids. Particularly in the LV DC grid, it is worth noting that most of the power electronics-interfaced appliances can be connected to the grid through DC/DC converters. As a result, the DC/AC module in conventional back-to-back converter for Wind Turbine (WT) system or cascaded inverter for Photovoltaic (PV) system can be omitted, with lower investment and higher efficiency. In addition to the functionality of voltage step-down, a ST combines the functionality of power conditioners, offering advanced features and various ancillary services to the grid, both in MV and LV side: improvement of the current and voltage profile [23], [24], balancing services [25], [26], and reliability improvement [8].

Control scenarios and associated communication are two essential elements for the ST-fed grid, which ensures a more reliable and resilient electric grid and better quality services [27]. As shown in Fig.1, the ST can host the data center which offers services to the grid. On the MV side, the ST regulates current absorption from the utility, delivering active power demanded from the loads in the LV grids. Due to the decoupling between the MV and the LV side, the nonactive power can be controlled to provide ancillary services like reactive power and harmonic compensation on the MV side while have ignorable influence on the LV side [26]. The ST can even supply active power during contingencies for instance in black start and in partial disconnection of the MV grid [28]. On the LV side, the ST controls the grid voltage and can offer miscellaneous services to the end customer. The amplitude and frequency are the two degrees of freedom of the ST LV side, which can be modified to interact with DERs and local loads aiming at having an influence on the power generation and consumption of the LV grid [29], [30]. For example, potential issues like overloading or reverse power flow can be solved by means of voltage frequency and amplitude control. Another possibility is to on-line identify the load sensitivity by applying the frequency and amplitude perturbation [31]. This service enables more accurate control actions for those control or services rely on load estimation. Moreover, the ST takes advantage of the power-electronics-based converters, alleviating the resonances in LV grid as a series active damper [32]. According to the control and communication requirements listed in Fig.1, MV and LV customers can flexibly subscribe services tailored by ST while have very limited impact on the 


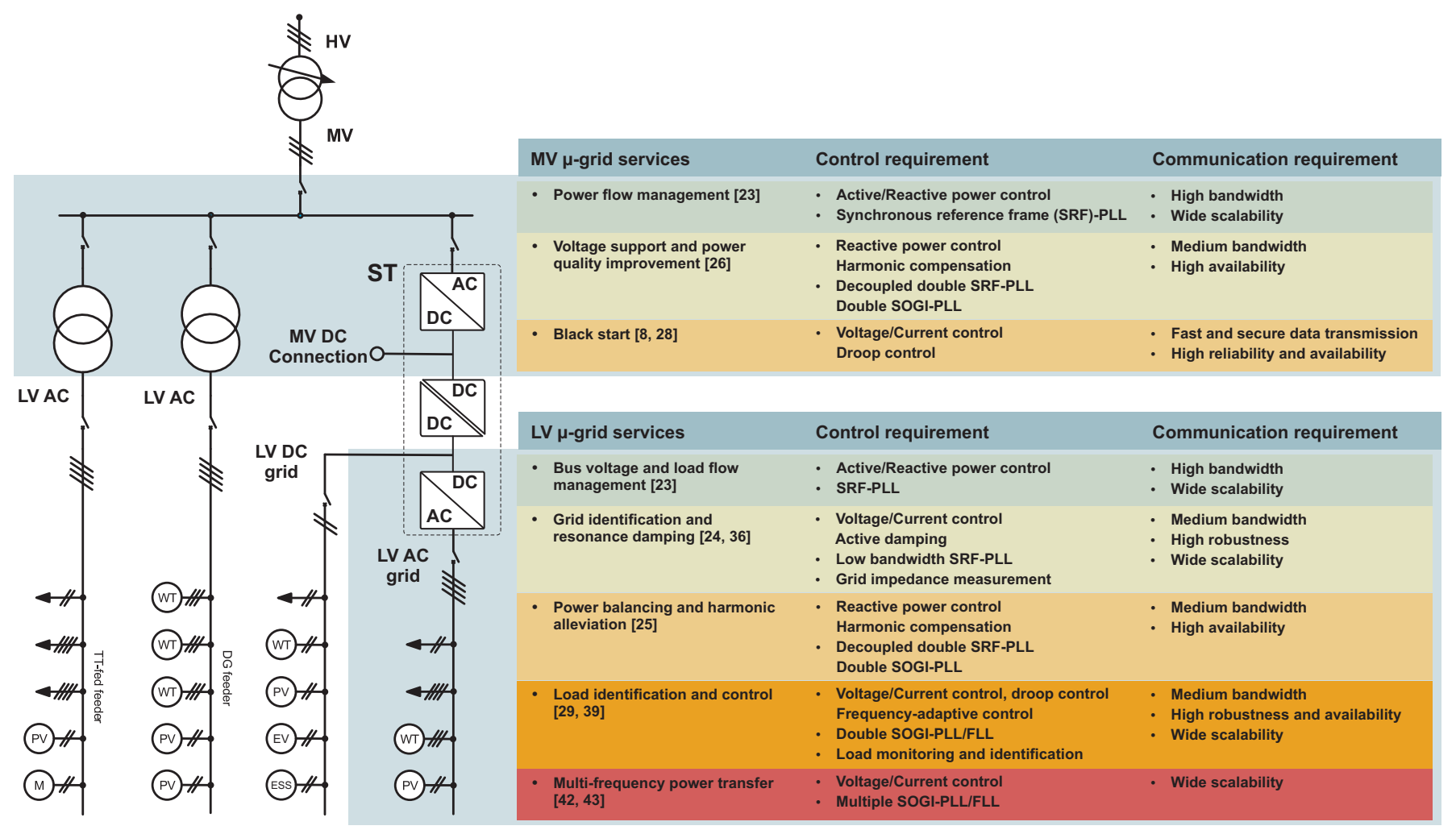

Fig. 1. ST-fed grid with associated services.

other feeders/grids. From the grid resilience perspective, these services effectively avoid the cascaded blackout, achieving a self-healing grid [33].

The ST is supposed to be the key component to emerge and manage all the available resources in terms of services, algorithms, and grid data within the ST-fed grid, and is able to allocate them to the distributed appliances in need or allow distributed appliances to access these shared resources. The virtual aggregation of resources is especially suitable for the services which rely on considerable data processing capacity and abundant grid information. It can avoid additional control and measurements implemented locally, which saves the cost and maintenance of local appliances. More importantly, these upcoming services enable the ST-fed grid to more rapidly adjust resources to meet the evolving and unpredictable requirements from utility and customers.

The advent of ST drives the control center delegating more functionalities to the lower level, so that 1) relieves the control burden of control center; 2) empowers the consumers with more choices and better services. The architecture of STfed grid is not either centralized or decentralized. It is semidecentralized since the ST interacts with the components of the grid and change its operation on the basis of the need.

\section{OPERATION OF A ST-FED GRID}

Selected control scenarios of ST-fed grid are reviewed in this section.

\section{A. Node Voltage and Power Flow Management}

One of the challenges posed by the penetration of renewables is the reverse power flow. As a result, overvoltage and current congestion would occur, causing the disconnection of DERs and potential damage to local appliances. In [23], this problem is addressed by equipping a ST with the capability of node voltage management. When a ST is associated to the DERs, the node voltage as well as current flow can be well regulated by curtailing the active and reactive power. Moreover, the ST allows the voltage and power profiles to be managed in per-phase mode, enabling the unbalanced power injection to the grid in case of the presence of unbalanced overload of MV grid, with voltage and current limit violations.

The system configuration and control structure are illustrated in Fig.2. The AC/DC power converters of ST are controlled by a power control outer loop and a current control inner loop with given active/reactive power set-points. An example of a power/current controller is shown in Fig.3, which is designed by using two cascaded synchronous controllers on $d q$ frame. Considering the MV bus voltages are symmetrical in most cases, the phase angle is acquired by a three-phase Synchronous Reference Frame Phase-Locked Loop (SRFPLL). A centralized controller is implemented in the data center (e.g. SCADA) of the substation to optimize the node voltage and power flow. Among various optimization schemes, the computation of node voltages and line currents sensitivity coefficients turns out to be a unique solution for a generic 


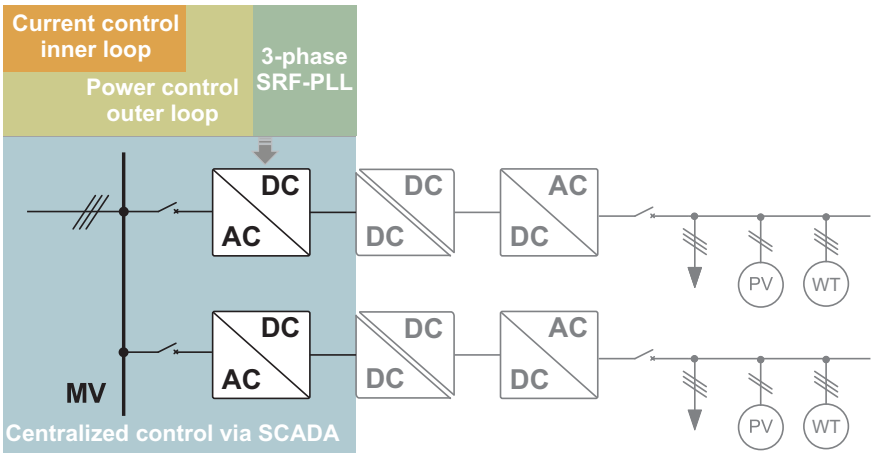

Fig. 2. Node voltage and power flow management by means of ST.

MV DC connection

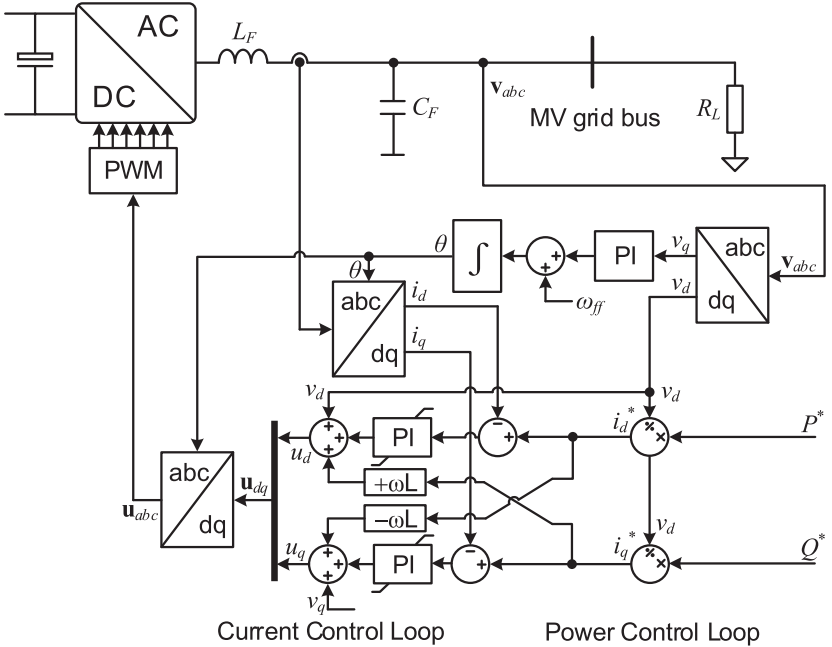

Fig. 3. Basic control structure of ST AC/DC converter during active/reactive power control.

unbalanced and radial electrical network, that is suitable for distribution grids [34]. The active/reactive power set-points of each ST hereby are computed by means of sensitivity coefficients computation process and disseminated though the communication network. To ensure real-time monitoring and computing process, a fast and reliable communication network with wide coverage distance is demanded.

\section{B. Voltage Support and Power Quality Improvement}

The ST is able to provide ancillary services like reactive power injection and power quality improvement to MV grid. The concept of "multi-function" ST has been proposed in [26] and several scenarios have been considered as well. In normal operation, the ST is expected to inject reactive power by using the surplus power generation of DERs so that it can 1) support and reduce the losses of MV grid; 2) block the reverse power flow to MV line. In case of IEEE 1459 events or disturbances, the ST is supposed to perform harmonic compensation and voltage support at the same time. The control system of this scenario is presented Fig.4. It is seen that the voltage support functionality can be achieved

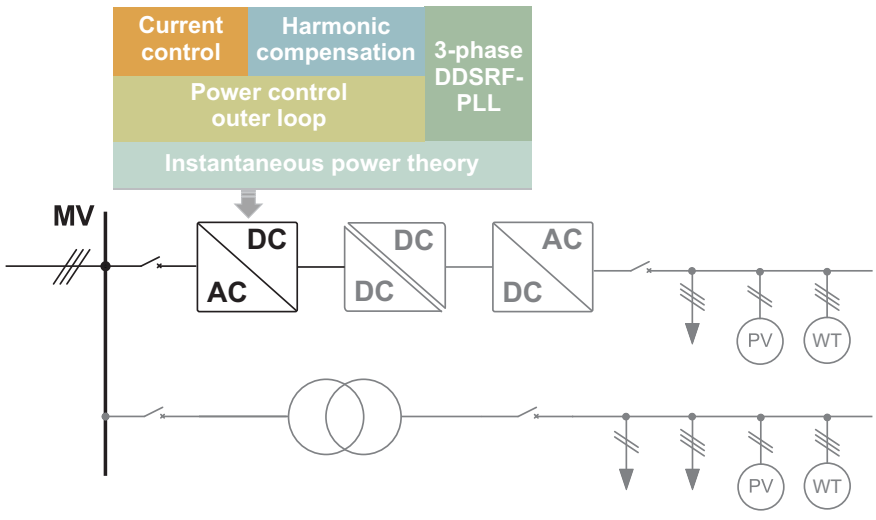

Fig. 4. Voltage support and power quality improvement by means of ST.

through reactive power control, whose control structure is similar to that of Fig.3. The power quality improvement on the other hand relies on the generalized instantaneous power theory which can calculate the accurate current setpoint for the purpose of harmonic compensation under generic grid conditions [35], and additional Harmonic Control (HC) schemes ensuring good harmonic current regulation. It is worth noting that an enhanced three-phase synchronization such as Decoupled Double SRF-PLL has to be used in this application to alleviate the undesirable features posed by grid faults or power quality issue.

In addition, a futuristic control scenario called dual microgrid operation is considered in [36]. The ST is able to become a grid-forming source in MV grid during MV faults or intentional disconnection for maintenance purpose. The AC/DC converter will switch from power/current control mode to voltage control mode so that it can provide stiff voltage to other feeders when the HV and MV breakers are switch off. As a result, the other feeders remain connecting to the grid by shedding non-critical loads. The scenario operation demands sufficient grid data during the transient stage to make sure the ST can fast and seamlessly take the responsibility of supporting the overall distribution grid. This scenario is particularly helpful to supply critical loads and black start procession.

\section{Grid Identification and Resonance Damping}

The massive integration of DERs poses resonances resulting compromised stability and power quality. In a ST-fed distribution grid, the ST offer the opportunity to identify and dampen the grid resonances [37], [38]. Seen from Fig.6, the ST LV side $\mathrm{DC} / \mathrm{AC}$ inverter can act as a grid impedance measurement and propose tailored active damping solutions. Depending on the characteristics of grid resonance, the ST can offer series active damping by using its DC/AC inverter or solicit one of the DERs providing shunt active damping. Usually, a multiloopbased active damping is used in ST LV side DC/AC to achieve series active damping, the typical control structure is shown in Fig.7. The damping factor of the LV grid is determined by the control coefficients of the inductance current inner 


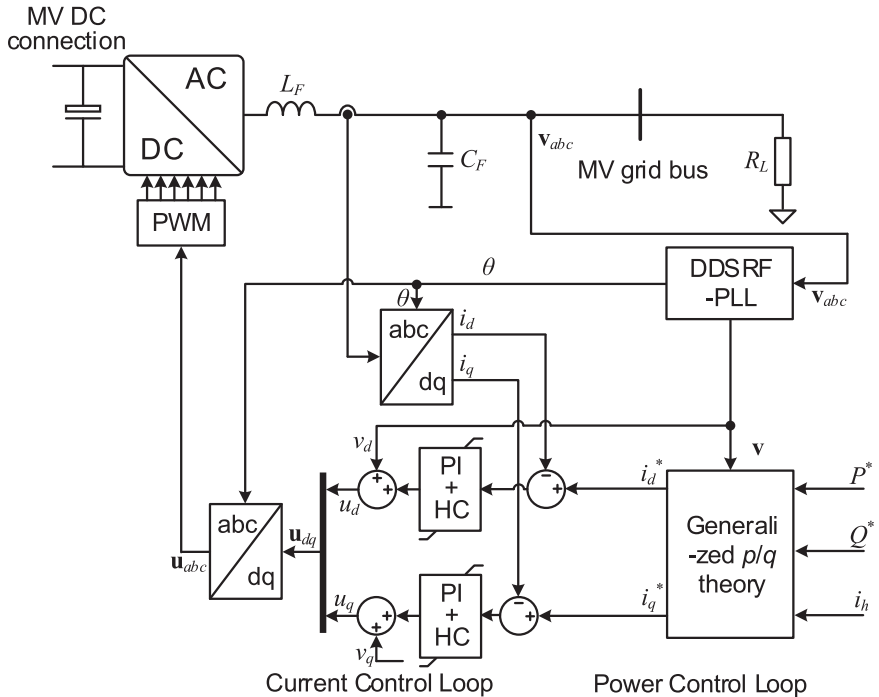

Fig. 5. Control structure of ST DC/AC converter for harmonic compensation.

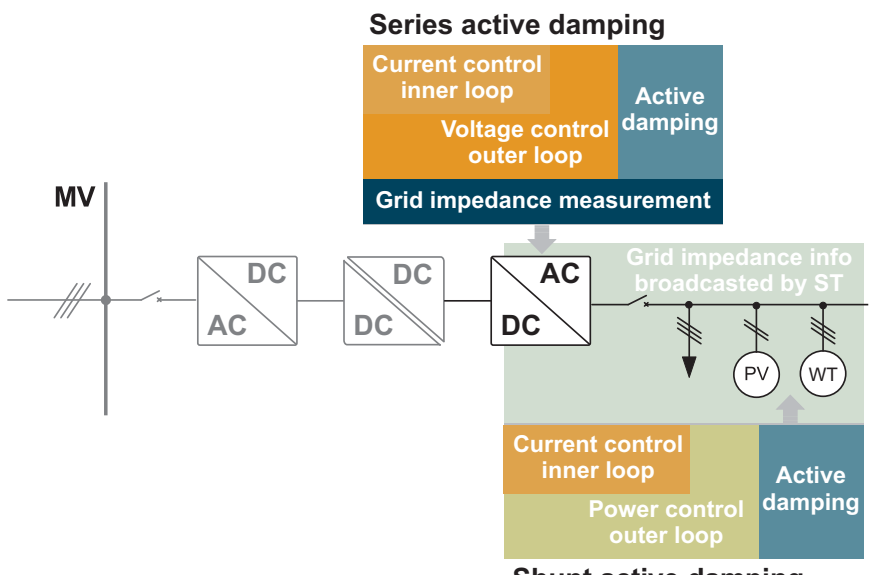

Fig. 6. Control structure for grid identification and resonance damping scenario.

loop. It is worth noting that the ST series damping is able to attenuate resonances within a wide range (e.g. between $0.1 \mathrm{kHz}$ to $10 \mathrm{kHz}$ ) and therefore benefits the vast majority of grid-interfaced appliances.

Unlike the series active damping, the shunt active damping is more likely to deal with the resonances in certain buses. The DER receives the resonance information which is identified and broadcast by the ST, utilizes customized filter-based damping methods to handle it. A study case of DER with the functionality of shunt active damping is presented in [39], where the notch filter active damping is utilized to mitigate the impedance resonance at certain frequency. Based on the acurrate impedance information, the DER can participate in resonance damping requiring no trial-and-error iterations. Due to the robustness of notch filter damping, the bandwidth of broadcasting network is not very stringent.

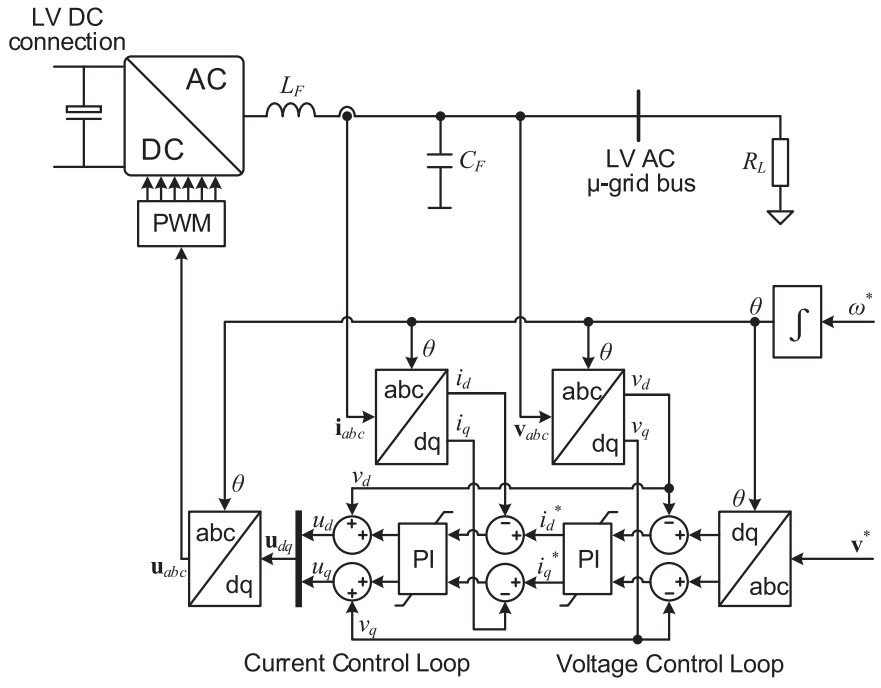

Fig. 7. Basic control structure of ST DC/AC converter.

\section{Load Identification and Control}

One of the advantageous aspects of ST is the decoupling between MV and LV grids thanks to the presence of DC links. It allows the ST to modify the LV side voltage waveform to intact with the DERs and loads with very limited impact on the MV grid. In [40], [29], two complementary control algorithms have been proposed to adapt the frequency and voltage to the power generation and consumption in order to achieve better power sharing and deal with some practical issue, such as overload and reverse power flow. Meanwhile, the load sensitivity should be identified to enable smooth operation during the LV frequency/voltage regulation. With these considerations, a combined load identification and control has been proposed and implemented through the ST [41]. The control structure of the ST as well as DERs for this scenario has been described in Fig.8. Based on the real-time load identification, the LV side frequency/voltage references are accordingly modified by following predefined curves, for example, the power/frequency curve. The control structure of Fig.7 can be used to regulated the LV side voltage. To inform local DERs, the frequency/voltage information will be broadcast by the ST to the LV grid. Following the frequency information, local DERs are thereby able to change power output by using droop control.

A further application on this topic is the frequency adaptive ST-fed distribution grid [30]. The before-mentioned frequency control is implemented in the ST CMU. A frequency adaptive repetitive control scheme along with a double Second-Order Generalized Integrator (SOGI)-PLL are adopted to maintain good voltage/current control performance under variable frequency condition. The proposed system provides a promising approach to address practical issue during load peaks and overcapacity of DERs. 


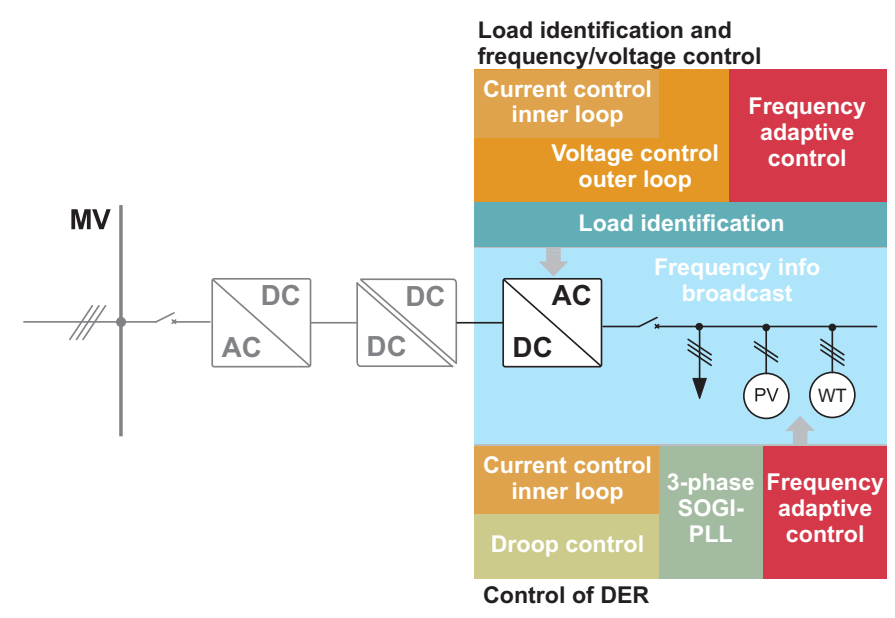

Fig. 8. Coordinated frequency and voltage overload control of ST-fed grid.

\section{E. Multi-Frequency Power Transfer}

The multi-frequency power transfer concept is derived from the frequency modulation in telecommunication where multiple frequencies are used to transmit information within a medium among several sender and receivers. In presence of ST, more than one frequency voltage/current waveforms can be modulated and used to send power from one point to another of the LV grid. Unlike the traditional distribution transformer, the "harmonic" frequency signals for power transfer has very limited impact on the ST lifetime, ST losses, and MV grid as well as MV breakers. In [42] and [43], fundamental frequency and third harmonic have been chosen as carrier frequencies in a ST-fed distribution grid to achieve the sophisticated power transfer. The basic control structure is shown in Fig.9, where the voltage control of Fig.7 has been used for LV side DC/AC inverter, while the power control of Fig. 3 is used for the grid-interfaced converters of DERs. In order to "decode" the power at different frequencies, multiple double SOGI Frequency-Locked Loops (SOGI-FLLs) are employed for frequency detection. Known the frequency, a SRF-PLL with the same characteristic frequency is utilized to detect the phase angle for each frequency. The combination of SOGIFLL and SRF-PLL facilities the accurate power transfer and at the same time eases the communication between sender and receiver.

\section{Communication Technologies of ST-Fed GRids}

The emerging of AMI which is used to measure, monitor, and analyze data facilitates the ST to have a comprehensive perspective on the grid, allowing ST to improve power management ability, power supply quality, and energy planning capability. Failures and events can be sensed through realtime monitoring and largely prevented by online diagnostics and protection, which improves the reliability and maintenance of the overall system. On the prerequisite of information exchange, a reliable, secure and interoperable communication network is the key element making the ST-fed grid concept a reality. A contemporary view of possible communication

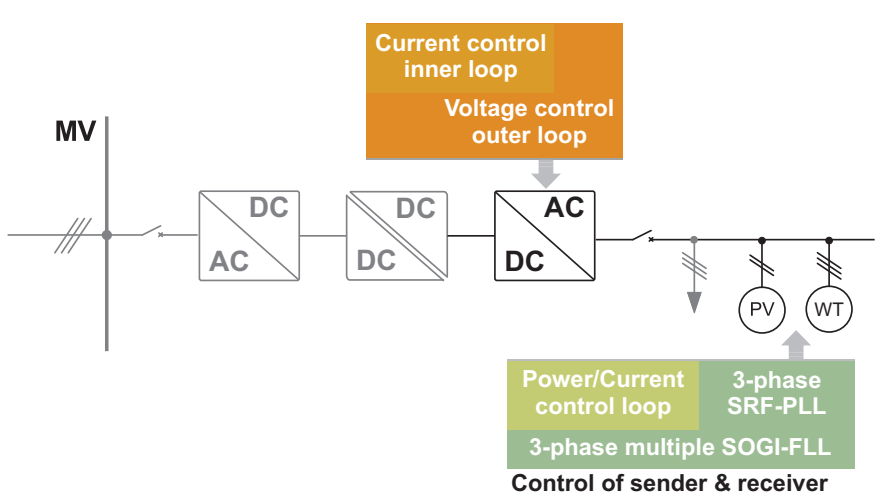

Fig. 9. Control structures of ST DC/AC converter and power sender as well as receiver in multi-frequency power transfer scenario.

technologies in ST-fed grid is given in the following section. The requirements and further challenges in terms of communication delay and loss are discussed as well.

\section{A. Possible Communication Architecture and Technologies}

Numerous architectures can be designed for different applications of ST-fed grid. The IEEE 2030-2011 standard which uses a hierarchical structure to provide guidelines for smart grid interoperability has been widely accepted in smart grid applications [44]. The IEEE 2030 standard defines guidelines for three hierarchical networks: private network, Wide Area Network (WAN), and core network, which are related to the customer properties, distribution domain, and power generation and transmission, respectively. In most applications, the ST locates at distribution domain (e.g. substations) facilitating information regulation in the WAN and data exchange between core network and private network as shown in Fig.10. Depending on the connected subnetworks, the end-points of a ST-fed grid can be an AMI in the Neighborhood Area Network (NAN) or a substation automation unit (e.g. Remote Terminal Unit (RMU) and Phasor Measurement Unit (PMU)) in the Field Area Network (FAN). It it worth noting the NAN and FAN mentioned here are two typical distribution domain networks which belong to the category of WAN. From the ST aspect, the logical representation of its lower layer network: private network, could be simply an AMI or smart meters. Nevertheless, because of the stringent grid codes or customized requirements, a small-scale or nano ST-fed grid could be set up, and normally the ST is demanded to be the only information interface to process and exchange data between local network and outer network. The best-known application is general hospital requiring high quality power supply for its medical facilities as well as apparatuses and can even be operated as a standalone grid in some emergency case. In these applications, the ST performs in a downstream private network and provides direct electricity and communication connection to the customer properties. Depending on the demands, the customized ST seamlessly contact each userends and communicate the data center or the ST in a up-level network. 


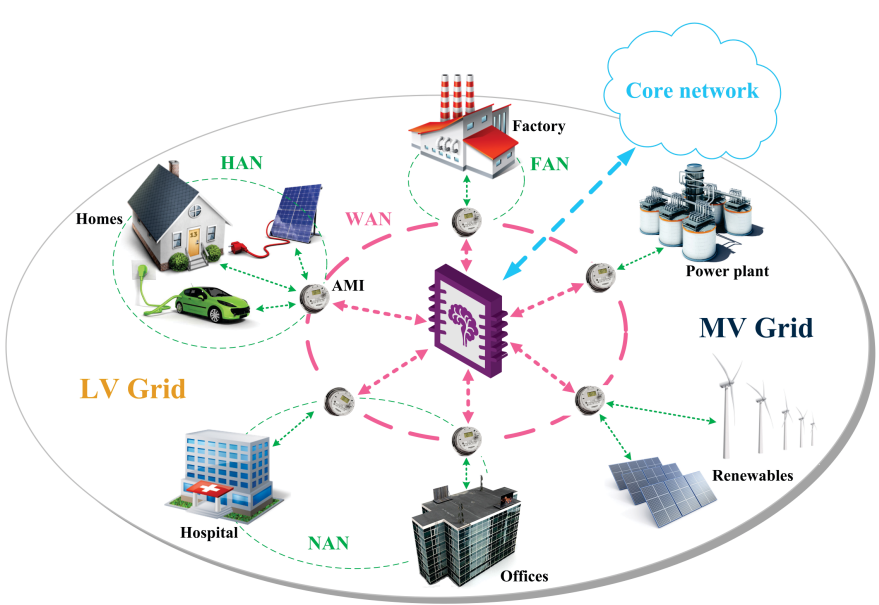

Fig. 10. Communication architecture of the ST-fed grid (the symbol in the center represents the ST).

The available communication technologies for smart grid have been reviewed in [45]. Similar to the smart grid, the majority of the available technologies can be used in a ST-fed grid depending on the control scenarios and communication architecture. Playing the role of a energy router, the ST is conjecture to not only manage and optimize the power flow but also regulate the information flow between different communication networks. As a result, various standard communication interfaces and protocols should be implemented in the ST enabling it to intact with the different types of data from smart meters and distributed appliances. Moreover, a data management system is necessary to endue a ST with the data storage and processing abilities.

According to the data transmission in Fig.10, the information flow in a ST-fed grid can be categorized as three types: the first flow is between different distributed appliances or AMI, e.g. data transmission between smart meters and DER, as suggested in literature [45], [46], PowerLine Communication (PLC) and local area wireless network such as the family of IEEE 802 network can be applied; the second kind of flow is between ST and local electric appliances as well as AMIs, and the third flow is between ST and core network or flow between two different STs (if multiple same level STs are applied in the grid/network). The latter two types of information flow requiring higher data transmission rate and wider coverage area, which can be accomplished by a WAN technology including cellular network and Digital Subscriber Line (DSL). A speculation of possible communication technologies in STfed grids is shown in TABLEI.

\section{B. Communication Challenges}

The benefits of ST-fed grids are based on the optimized control scenarios and the supporting of communication infrastructure. Although the reliability of the communication network is relatively high thanks to the progressing technology, it still could involve the performance degradation due to various factors. One significant factor is the increasing of DERs and smart meters, which impose the network a large number of nodes and repeatedly access the network with burst of data. In the foreseeable future, the rate of information throughput in a ST-fed grid grows in geometric progression and will further severe the control burden of information flow. As a consequence, the random transmission failure or uncertainty in terms of data congestion, data drop and delay (jitter) will increase as well. These impacts have to be taken into account in the plan and design of a ST-fed grid to make sure the grid resilience. Especially in centralized control scenarios, the transmission of set-points is crucial. The inappropriate setpoints not only deteriorate the control performance but also jeopardize the safety of the whole system. For example, the influence of communication delay and loss in a frequency controlled microgrid has been investigated in [22]. The results show that although the microgrid can overcome a noticeable delay (e.g. $2 \mathrm{~s}$ ) of frequency set-points, it is very likely to collapse with higher data losses. While in a decentralized grid, it is known that the grid can perform acceptable performance with the communication deterioration. It is one of the reasons that advocates of distributed control envisage a shift from the centralized control to decentralized control of grids in the very near future. However, even in this premise, the effectiveness and service grade become dim and the vast of advantages provided by the communication are gone off.

With the advent of ST, advanced services can be offered to better use the exciting communication resources. For instance, Quality of Service (QoS), a technology derived from telephony or computer network [52], can be embedded in the ST data management to better support and optimize the communication network as well as the grid. Generally speaking, the QoS mechanism requires the ST to define the priorities of various types of information based on their importance to the grid, including contribution to system stability, power quality, energy demand, etc. During the period of data congestion, this service enables the information flow with higher priority to have a wider transmission bandwidth and smoother transmission quality, but at the same time sacrifices the quality of other information with lower priority. As a result, a tradeoff has to be made between the performance of ST-fed grid and the service quality of communication network. Despite it cannot work out the grid's full potential, it is the best means of grid optimization with limited communication capability.

In addition, a backup communication-less system or redundant communication network for critical section could be an alternative to improve the resilience of a ST-fed grid. As shown in [21], a optimal redundant communication link has been designed based on the directed graph theory. Because of the sparest property, each node in the communication network can maintain graphical connectivity in case of partial failure. In the communication-critical grid, for example the frequency adaptive ST-fed distribution grid of Section III-D, the lost of frequency set-points could lead to power imbalance and voltage instability. A reserved communication-less alternative by using the SOGI-FLL has been proposed to assist the local controller to correct its power/frequency set-points under data loss condition. A minor (less than $50 \mathrm{~ms}$ ) detection delay 
TABLE I

Available Communication Technologies IN ST-Fed GRID

\begin{tabular}{|c|c|c|c|c|}
\hline Technology & Standard & Data Rate & Distance & Network \\
\hline \multirow{3}{*}{ PLC [47] } & Ultra Narrow Band (UNB)-PLC & $100 \mathrm{bps}$ & $150 \mathrm{~km}$ or more & core network \\
\hline & Narrow Band (NB)-PLC & $10-500 \mathrm{kbps}$ & $10 \mathrm{~km}$ or more & WAN \\
\hline & Broad Band (BB)-PLC & $1-200 \mathrm{Mbps}$ & $1 \mathrm{~km}$ & private network (e.g. HAN, FAN, NAN) \\
\hline \multirow{4}{*}{ DSL [48] } & High-bit-rate DSL (HDSL) & $1.5-2 \mathrm{Mps}$ & $1.6-18 \mathrm{~km}$ & private/WAN \\
\hline & Asymmetric DSL (ADSL) & 1.5-24 Mbps down/ 0.5-3.5 Mbps up & up to $5.5 \mathrm{~km}$ & private network \\
\hline & Symmetric DSL (SDSL) & $256 \mathrm{kbps}-2.3 \mathrm{Mbps}$ & up to $3 \mathrm{~km}$ & private network \\
\hline & Very-high-bit-rate DSL (VDSL) & 55-100 Mbps down/ 3-100 Mbps up & $300 \mathrm{~m}$ & private network \\
\hline \multirow{2}{*}{ Wireless Mesh [49] } & IEEE 802.11 & 6-54 Mbps & $0.1-5 \mathrm{~km}$ & private/WAN \\
\hline & IEEE 802.15 & $11 \mathrm{Mbps}-5.3 \mathrm{Gbps}$ & $20-100 \mathrm{~m}$ & private network \\
\hline \multirow{4}{*}{ Cellular [50], [51] } & $2.5 \mathrm{G}$ & 40-500 kbps down/ 9.6-42.8 kbps up & up to $200 \mathrm{~km}$ & WAN/core network \\
\hline & $3 \mathrm{G}$ & $200 \mathrm{kbps}-2 \mathrm{Mbps}$ & $10-72 \mathrm{~km}$ & WAN \\
\hline & $3.5 \mathrm{G}$ & 14-337 Mbps down/ 5.76-34 Mbps up & $5-20 \mathrm{~km}$ & private/WAN \\
\hline & $4 \mathrm{G}$ & 100 Mbps-1 Gbps down/ 50-500 Mbps up & $3-30 \mathrm{~km}$ & private/WAN \\
\hline
\end{tabular}

occurs but is negligent to the droop control, allowing DER to output desired power even the communication is completely lost.

\section{Conclusions}

The increasing penetration of renewables poses new challenges and research issues on the power grids. This paper has highlighted a promising approach, the ST-fed grid, to address the coming up issues and enhance the grid capability in terms of hosting capacity, efficiency, reliability, resilience, and safety. To make this futuristic electric network a reality, communication and control scenarios have been proposed, which are exploiting new opportunities and potentials of the existing power system.

In this paper, the ancillary services provided by the STfed grid are introduced and both control and communication requirements are described. The control scenarios and communication technologies for the ST-fed grids have been discussed. Since the ST-fed grid opens a new frontier, the state-of-theart and research challenges have been presented. To solve the challenges, future work on this subject includes definition of services, design of control architectures, and design of robust control considering communication failures.

\section{ACKNOWLEDGMENT}

The research leading to these results has received funding from the European Research Council under the European Union's Seventh Framework Programme (FP/2007-2013) / ERC Grant Agreement n. [616344] - HEART.

\section{REFERENCES}

[1] "Federal Ministry for Economic Affairs and Energy Historic data about the development of renewable energies in Germany," http://http://www.erneuerbare-energien.de/.

[2] V. Quezada, J. Abbad, and T. Roman, "Assessment of energy distribution losses for increasing penetration of distributed generation," IEEE Transactions on Power Systems, vol. 21, no. 2, pp. 533-540, May 2006.

[3] E. Coster, J. Myrzik, B. Kruimer, and W. Kling, "Integration issues of distributed generation in distribution grids," Proceedings of the IEEE, vol. 99, no. 1, pp. 28-39, Jan 2011.
[4] A. Bhowmik, A. Maitra, S. Halpin, and J. Schatz, "Determination of allowable penetration levels of distributed generation resources based on harmonic limit considerations," IEEE Transactions on Power Delivery, vol. 18, no. 2, pp. 619-624, April 2003.

[5] "Harmonic Identification, Mitigation and Control in Power Electronics based Power System," http://harmony.et.aau.dk/.

[6] L. Harnefors, M. Bongiorno, and S. Lundberg, "Input-admittance calculation and shaping for controlled voltage-source converters," IEEE Transactions on Industrial Electronics, vol. 54, no. 6, pp. 3323-3334, Dec 2007.

[7] X. Wang, F. Blaabjerg, and W. Wu, "Modeling and analysis of harmonic stability in an ac power-electronics-based power system," IEEE Transactions on Power Electronics, vol. 29, no. 12, pp. 6421-6432, Dec 2014.

[8] A. Q. Huang, M. L. Crow, G. T. Heydt, J. P. Zheng, and S. J. Dale, "The future renewable electric energy delivery and management (freedm) system: The energy internet," Proceedings of the IEEE, vol. 99, no. 1, pp. 133-148, Jan 2011.

[9] R. H. Lasseter, J. H. Eto, B. Schenkman, J. Stevens, H. Vollkommer, D. Klapp, E. Linton, H. Hurtado, and J. Roy, "Certs microgrid laboratory test bed," IEEE Transactions on Power Delivery, vol. 26, no. 1, pp. 325332, Jan 2011.

[10] M. Z. Kamh, R. Iravani, and T. H. M. EL-Fouly, "Realizing a smart microgrid - pioneer canadian experience," in Power and Energy Society General Meeting, 2012 IEEE, July 2012, pp. 1-8.

[11] H. Farhangi, "The path of the smart grid," IEEE Power and Energy Magazine, vol. 8, no. 1, pp. 18-28, January 2010.

[12] "The Highly Efficient And Reliable smart Transformer (HEART)," http://heart.tf.uni-kiel.de/en/.

[13] A. L. Dimeas and N. D. Hatziargyriou, "Operation of a multiagent system for microgrid control," IEEE Transactions on Power Systems, vol. 20, no. 3, pp. 1447-1455, Aug 2005.

[14] "DESL low voltage microgrid," http://desl-pwrs.epfl.ch/microgrid, accessed: 2014-03-12

[15] A. Bernstein, L. Reyes-Chamorro, J.-Y. Le Boudec, and M. Paolone, "A composable method for real-time control of active distribution networks with explicit power setpoints. part i: Framework," Electric Power Systems Research, vol. 125, pp. 254-264, 2015.

[16] R. G. Smith, "The contract net protocol: High-level communication and control in a distributed problem solver," IEEE Transactions on Computers, vol. C-29, no. 12, pp. 1104-1113, Dec 1980.

[17] S. Tatikonda and S. Mitter, "Control under communication constraints," IEEE Transactions on Automatic Control, vol. 49, no. 7, pp. 1056-1068, July 2004.

[18] N. Cohn, "Recollections of the evolution of realtime control applications to power systems," Automatica, vol. 20, no. 2, pp. 145 - 162, 1984.

[19] M. C. Chandorkar, D. M. Divan, and R. Adapa, "Control of parallel connected inverters in standalone ac supply systems," IEEE Transactions on Industry Applications, vol. 29, no. 1, pp. 136-143, Jan 1993. 
[20] W. Ren, R. W. Beard, and E. M. Atkins, "Information consensus in multivehicle cooperative control," IEEE Control Systems, vol. 27, no. 2, pp. 71-82, April 2007.

[21] V. Nasirian, S. Moayedi, A. Davoudi, and F. L. Lewis, "Distributed cooperative control of dc microgrids," IEEE Transactions on Power Electronics, vol. 30, no. 4, pp. 2288-2303, April 2015.

[22] G. Strbac, N. Hatziargyriou, J. P. C. Lopes, C. Moreira, A. Dimeas, and D. Papadaskalopoulos, "Microgrids: Enhancing the resilience of the european megagrid," IEEE Power and Energy Magazine, vol. 13, no. 3, pp. 35-43, May 2015.

[23] G. De Carne, M. Liserre, K. Christakou, and M. Paolone, "Integrated voltage control and line congestion management in active distribution networks by means of smart transformers," in IEEE 23rd International Symposium on Industrial Electronics (ISIE), June 2014, pp. 2613-2619.

[24] Z.-X. Zou, M. Liserre, Z. Wang, M. Cheng, and S. Fan, "Resonance damping in a smart transformer-based microgrid," in 41st Annual Conference of the IEEE Industrial Electronics Society (IECON), Nov 2015, pp. 956-964.

[25] G. De Carne, G. Buticchi, M. Liserre, C. Yoon, and F. Blaabjerg, "Voltage and current balancing in low and medium voltage grid by means of smart transformer," in IEEE Power Energy Society General Meeting, July 2015, pp. 1-5.

[26] C. Kumar and M. Liserre, "Operation and control of smart transformer for improving performance of medium voltage power distribution system," in IEEE 6th International Symposium on Power Electronics for Distributed Generation Systems (PEDG), June 2015, pp. 1-6.

[27] M. Liserre, G. Buticchi, M. Andresen, G. D. Carne, L. F. Costa, and Z. X. Zou, "The smart transformer: Impact on the electric grid and technology challenges," IEEE Industrial Electronics Magazine, vol. 10, no. 2, pp. 46-58, Summer 2016.

[28] C. Kumar, Z. Zou, and M. Liserre, "Smart transformer-based hybrid grid support to loads in partial disconnection of mv/hv power system," in IEEE Energy Conversion Congress \& Expo (ECCE), Sept. 2016, accepted.

[29] G. De Carne, G. Buticchi, M. Liserre, P. Marinakis, and C. Vournas, "Coordinated frequency and voltage overload control of smart transformers," in IEEE PowerTech, June 2015, pp. 1-5.

[30] Z.-X. Zou, G. De Carne, G. Buticchi, and M. Liserre, "Frequency adaptive control of a smart transformer-fed distribution grid," in IEEE Applied Power Electronics Conference (APEC), March 2016, pp. 3493 3499.

[31] G. D. Carne, M. Liserre, and C. Vournas, "On-line load sensitivity identification in lv distribution grids," IEEE Transactions on Power Systems, pp. 1-2, 2016. [Online]. Available: $\mathrm{http}$ ://ieeexplore.ieee.org/stamp/stamp.jsp?tp=\&arnumber=7493702\&isn umber $=4374138$

[32] X. Wang, Y. Pang, P. C. Loh, and F. Blaabjerg, "A series-lc-filtered active damper with grid disturbance rejection for ac power-electronics-based power systems," IEEE Transactions on Power Electronics, vol. 30, no. 8, pp. 4037-4041, Aug 2015.

[33] K. Moslehi, A. B. R. Kumar, D. Shurtleff, M. Laufenberg, A. Bose, and P. Hirsch, "Framework for a self-healing power grid," in IEEE Power Engineering Society General Meeting, June 2005, pp. 3027 Vol. 3-.

[34] K. Christakou, J. Y. LeBoudec, M. Paolone, and D. C. Tomozei, "Efficient computation of sensitivity coefficients of node voltages and line currents in unbalanced radial electrical distribution networks," IEEE Transactions on Smart Grid, vol. 4, no. 2, pp. 741-750, June 2013.

[35] F. Z. Peng and J.-S. Lai, "Generalized instantaneous reactive power theory for three-phase power systems," IEEE Transactions on Instrumentation and Measurement, vol. 45, no. 1, pp. 293-297, Feb 1996.

[36] C. Kumar and M. Liserre, "A new prospective of smart transformer application: Dual microgrid (dmg) operation," in 41 st Annual Conference of the IEEE Industrial Electronics Society, Nov 2015, pp. $004482-$ 004487.

[37] F. Hahn, S. Brüske, F. W. Fuchs, B. Benkendorff, and M. Liserre, "Wide frequency range medium-voltage grid impedance analysis by current injection of a multi-mw power converter," in 18th European Conference on Power Electronics and Applications (EPE), Sept. 2016, accepted.

[38] M. Liserre, L. Jessen, Z.-X. Zou, B. Benkendorff, and F. Fuchs, "Resonance identification and damping in ac-grids by means of grid converters," in 42nd Annual Conference of the IEEE Industrial Electronics Society (IECON), Oct 2016.

[39] R. P. na Alzola, M. Liserre, F. Blaabjerg, M. Ordonez, and T. Kerekes, "A self-commissioning notch filter for active damping in a three-phase lcl - filter-based grid-tie converter,' IEEE Transactions on Power Electronics, vol. 29, no. 12, pp. 6754-6761, Dec 2014.

[40] G. Buticchi, M. Liserre, D. Barater, C. Concari, A. Soldati, and G. Franceschini, "Frequency-based control of a micro-grid with multiple renewable energy sources," in IEEE Energy Conversion Congress and Exposition (ECCE), Sept 2014, pp. 5273-5280.

[41] G. De Carne, G. Buticchi, M. Liserre, and C. Vournas, "Load identification and control by means of smart transformer," IEEE Transactions on Power Electronics, 2016, accepted.

[42] S. Brüske, G. D. Carne, and M. Liserre, "Multi-frequency power transfer in a smart transformer based distribution grid," in 40th Annual Conference of the IEEE Industrial Electronics Society (IECON), Oct 2014, pp. 4325-4331.

[43] S. Brüske, G. Buticchi, and M. Liserre, "Control strategies for multifrequency power transfer in a smart-transformer-fed low-voltage grid," in IEEE 13th International Conference on Industrial Informatics (INDIN), July 2015, pp. 1576-1582.

[44] Y. Kabalci, "A survey on smart metering and smart grid communication," Renewable and Sustainable Energy Reviews, vol. 57, pp. 302-318, 2016.

[45] V. C. Gungor, D. Sahin, T. Kocak, S. Ergut, C. Buccella, C. Cecati, and G. P. Hancke, "Smart grid technologies: Communication technologies and standards," IEEE Transactions on Industrial Informatics, vol. 7, no. 4, pp. 529-539, Nov 2011.

[46] W. Luan, D. Sharp, and S. Lancashire, "Smart grid communication network capacity planning for power utilities," in Transmission and Distribution Conference and Exposition, 2010 IEEE PES, April 2010, pp. 1-4.

[47] S. Galli, A. Scaglione, and Z. Wang, "For the grid and through the grid: The role of power line communications in the smart grid," Proceedings of the IEEE, vol. 99, no. 6, pp. 998-1027, June 2011.

[48] J. W. Lechleider, "High bit rate digital subscriber lines: a review of hdsl progress," IEEE Journal on Selected Areas in Communications, vol. 9, no. 6, pp. 769-784, Aug 1991.

[49] H. Gharavi and B. Hu, "Multigate communication network for smart grid," Proceedings of the IEEE, vol. 99, no. 6, pp. 1028-1045, June 2011.

[50] D. M. Laverty, D. J. Morrow, R. Best, and P. A. Crossley, "Telecommunications for smart grid: Backhaul solutions for the distribution network," in Power and Energy Society General Meeting, July 2010, pp. 1-6.

[51] P. P. Parikh, M. G. Kanabar, and T. S. Sidhu, "Opportunities and challenges of wireless communication technologies for smart grid applications," in Power and Energy Society General Meeting, July 2010, pp. 1-7.

[52] A. Menychtas, D. Kyriazis, and K. Tserpes, "Real-time reconfiguration for guaranteeing qos provisioning levels in grid environments," Future Generation Computer Systems, vol. 25, no. 7, pp. 779 - 784, 2009. 